\title{
Tanulmány
}

\section{Amani Mejri \\ The interpretation of English reflexive and non-reflexive pronouns by Tunisian students}

\begin{abstract}
This study addresses English anaphor interpretation by Tunisian high school students. Along with seeking to obtain a preliminary view on this specific issue of second language acquisition, this pilot study equally aims at exploring possible similarities and differences in the interpretation of pronouns between Tunisian-Arabic native speakers and English speakers in previous child language acquisition studies. Notably, this study aims at examining whether Tunisian speakers are accurate in the interpretation of reflexives and whether they face any issues in pronoun interpretation. In a questionnaire elaborated in line with these motives, 24 Tunisian high school students interpreted some constructions including reflexive and non-reflexive pronouns. Their performance on the English pronominals, introduced within some constructions such as PNPs and PPs, showed some variation patterns when interpreting pronouns and reflexives as well. It was recorded that their pronoun interpretation in this pilot study was similar to L1 speakers, given that the Tunisian participants were accurate to some extent in their interpretation of reflexives and less accurate with pronouns. However, their pronoun interpretation, marked by its variation, could not be indicative of their grammatical competence as it is an introductory schema of the way they approach reflexives and non-reflexives. The pronominal difference between the two researched languages is underscored. Yet attributing the participants' pronoun interpretation to this pronominal difference could not be maintained, as it is not confirmed through this pilot study.

Keywords: English anaphor, Principle A, Principle B, computational complexity, Delay in Principle B Effect, second language acquisition, child language
\end{abstract}

\section{Introduction}

Claims from child language research concerning pronoun acquisition constitute a matter of investigation in second language research as well. First language acquisition hypotheses concern L1 speakers' performance when reflexive and non-reflexive pronouns are being interpreted. In fact, L1 speakers' performance on reflexives, particularly that of children between the age of 3 and 6 years old, is accurate and considered as adult-like performance (Chien \& Wexler 1990). Nevertheless, children of the same age still face some problems in interpreting non-reflexive pronouns (Thornton \& Wexler 1999). This performance is accounted for as an exemplification of children's knowledge of the locality condition, when reflexives are under scrutiny, stipulating that a reflexive pronoun is a bound variable (Conroy, Takahashi, Lids, \& Philips 2009; Thornton \& Wexler 1999). Concerning pronouns, children's performance reflects their pragmatic knowledge deficiency at this particular stage of language 
acquisition (Chien \& Wexler 1990), while for other researchers it reflects a computational load due to the limited capacity of children's working memory conducive to a delay in pronoun interpretation (Grodzinsky \& Reinhart 1993; Reinhart 2001). The fact that pronoun acquisition is delayed when compared to reflexives represents the substance for an ongoing debate given that it is associated with pragmatic deficiency for Chien and Wexler (1990), while it is a matter of computation for Reinhart $(2011,2006)$.

This research intersection between L1 and L2 language acquisition has led different researchers to draw patterns of similarities in the way anaphors are interpreted by L1 and L2 speakers (Slabakova et al. 2017). Other researchers, such as White (1998) postulate that the same interpretation issues, whether computational or pragmatic, could not be part of pronoun interpretation in second language contexts. In this paper, the aim is to investigate whether there is any discrepancy between pronoun and reflexive pronoun interpretation in a second language context and to compare it to L1 research prior to charting the reasons of any possible similarities or differences. In this setting, Tunisian Arabic is the native language of the participants, which is an under-researched language compared to English. Based on the pronominal difference between both languages, the primary motive is to obtain a general view on this L1 context, and to examine English pronoun interpretation patterns for Tunisian speakers.

To address this topic, this research paper covers an introductory theoretical background followed by the study-related information and discussion. The first part of this article addresses the acquisition of pronouns and reflexives in child language research, notably it surveys some studies in this research area. The subsequent section is devoted to L2 pronoun interpretation. Particular focus is attributed to the widely studied reflexives, plus introducing some studies that investigated pronoun interpretation cross-linguistically. Prior to reporting the current study methodology, findings and discussion, the last part of the theoretical background tackles the variation that punctuates pronoun interpretation in L1 and L2 acquisition. It similarly surveys the active variables that intervene in pronoun interpretation in the course of L1 and L2 acquisition.

\section{Binding Theory: Principle $A$ and Principle $B$ in child language acquisition}

\subsection{Binding Theory: Principle A and $B$}

The interpretation of noun phrases is regulated by diverse structural requirements under the Binding Theory. Anaphors and pronouns, as two different pronominal types, are regulated by Principle A and Principle B, respectively;

- Principle A: An anaphor is bound in its local domain

- Principle B: A pronoun is free in its local domain (Hamman 2011).

As regards anaphor processing, their antecedent has to be part of their local domain, while pronouns are free within the same local domain, as shown in the following examples:

1. a. Mary $y_{i}$ washed herself $f_{i}$

b. Mary $\mathrm{i}_{\mathrm{i}}$ washed her

In the example 1(a), the reflexive pronoun herself is bound by the subject Mary. In the example 1(b), the pronoun her is not bound by the subject Mary. Pronouns are free in their 
local domains. Where pronouns are free, anaphors are bound. They are in complementary distribution (Chomsky 1986).

In considering pronoun interpretation, Principle B blocks constructions such as 2, should the pronoun him be bound by its clause-mate antecedent Max:

2. Max $_{\mathrm{i}}$ criticizes himself $_{\mathrm{i} / \text { him }_{\mathrm{i}} *}$

The domain that specifies where a reflexive is bound is where the non-reflexive pronoun is free. Hence, the pronoun is free in its local domain (Hargeman 1994). Specifically, the pronoun gets its value assigned outside the local domain, unlike reflexives.

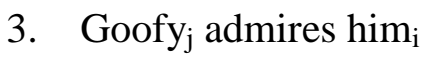

In (3), both the NP Goofy and the pronoun him have distinct indices and the pronoun is interpreted exophorically, that is based on the context and not having an intra-sentential antecedent (Guasti 2012).

\subsubsection{Long-distance anaphors: PNP and PP constructions}

As a revision to anaphor binding in the classical Binding Theory, Reinhart and Reuland (1993) postulate that anaphors can be linked to an antecedent outside of their local domain. Within some constructions such as picture NPs and PPs, anaphors can be linked to an antecedent that is not part of their local domain. This is explicated through the notion of predication upon which Reinhart and Reuland (1993) based their analysis of anaphor distribution.

4. Mary $\mathrm{y}_{\mathrm{i}}$ washed herself $\mathrm{i}_{\mathrm{i}}$

In 4, the verb wash is a transitive predicate allowing one if its arguments to be coindexed with its antecedent. In this effort, Reinhart and Reuland define their notion of syntactic predicates, having their arguments realized and $\theta$-roles as well as case assigned. The verb in 4 is an example of a syntactic predicate since its grammatical structure is realized by its arguments (the reflexive pronoun+ the antecedent). To define the semantic predicates, the researchers state that the arguments are rather licensed by the lexicon and the subject is not required, unlike the syntactic predicates (Reinhart \& Reuland 1993). NP and PP constructions are exemplifications of these predicates as in 5:

5. a. Lucie L saw a picture of herself $_{i}$

b. Lucie $_{i}$ saw a pricture of herself $f_{i} /$ her $_{i}$

To elucidate, anaphors in NP constructions can be logophoric in their interpretation. This means that they are either long-distance anaphors or exempt anaphors. Their interpretation is based on the context rather than the syntactically defined local domain (Reinhart \& Reuland 1993).

6. $\mathrm{John}_{\mathrm{i}}$ saw a picture of himself $\mathrm{i} / \operatorname{him}_{\mathrm{i}}$ 
In 6, John and himself are not coarguments of the verb, as John is the subject of the verb, while the reflexive pronoun is part of the VP internal argument. In this construction, the N picture is the predicate of the NP and the anaphor is its internal argument. The N picture is a semantic predicate. Given that John and himself are coindexed, the anaphor in this regard is subject to long-distance binding. This instance of long-distance binding allows to some extent the substitution of the reflexive by a pronoun (Asuder \& Keller 2001). This is motivated by some pragmatic reasons, rather than structural constraints.

Another occurrence of anaphors when they have logophoric properties is the PP construction.

7. a. Mary talks to herself $_{\mathrm{i}}$

b. Mary $y_{i}$ saw a snake near herself ${ }_{i} /$ her $_{i}$

The two constructions 7(a) and (b) are different in terms of the type of the prepositions introduced; while in 7(a) the preposition is a role selector of the verb and does not form a predicate, the preposition near in 7(b) does form a semantic predicate. In 7(a), the pronoun and the antecedent are coindexed and the anaphor does not have logophoric properties, hence they are both coarguments and the preposition is a role selector for the verb (Reinhart \& Reuland, 1993). In 7(b), the antecedent and the anaphor are not coarguments of the verb, unlike 7(a). The anaphor is an argument of the PP construction and it is a long-distance anaphor. In this example, pronoun resolution is allowed (Asudeh \& Keller 2001) given that the long-distance binding is not achieved through coargument coindexation (Reinhart \& Reuland 1993).

\subsubsection{Pronoun coreference}

Principle B clearly indicates that pronouns cannot be bound by an intra-sentential antecedent in their clause domain (Haegeman 1994). They can, however, be coreferential with such an antecedent under special conditions. Binding and coreference are different in the sense that binding is dictated by absolute syntactic conditions, whereas coreference is relative and context-oriented (Reinhart 2006). In constructions such as 8(a) and (b), the difference can be further explicated.

8. a. Oscar $_{\mathrm{i}}$ loves himi $_{\mathrm{i}}$

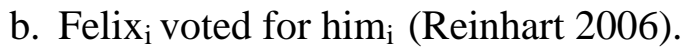

In constructions similar to 8 , the pronoun is referentially linked to a c-commanding NP, meaning that the pronoun is anaphorically interpreted (Guasti 2012). The reading available through coreference shows that only Oscar in 8(a) loves Oscar and no one else does. The same reading is also true for 8(b), as only Felix voted for Felix and no one else voted for Felix. Under the binding reading, however, it should be indicated that Felix voted for himself and everyone else voted for their own selves. As Reinhart (2006) conceptualizes it, then, the coreference reading needs to be distinguishable from the binding reading to be licensed. The coreference reading of 8(a) and (b) is motivated by context and is distinguishable from the binding reading that is motivated by syntactic constraints. To evaluate the licit reading, both representations of the coreference and binding are maintained and computed against context. 
This is a process of reference-set computation aiming at an effort of a semantic disambiguation based on the context that motivates the coreference readings vs. the binding one (Reinhart 2006).

\subsection{Child language acquisition: Reflexives vs. pronouns}

The functioning as well as the processes of these abstract NP interpretation tools are under scrutiny within language acquisition research with the varied performance patterns of children on Principle A and Principle B. Different experiments reported results on children's interpretation of anaphors indicating their advanced comprehension when it comes to indentifying the antecedent of a reflexive and showing their awareness of the locality condition regulating reflexive pronouns (Elbourne 2005; Hamann 2011; Conroy et al. 2009). Experimental methodologies used to test children's linguistic knowledge of Principle A are varied from truth judgement tasks to act-out tasks, among others (Chien \& Wexler 1990). Yet although methodologies are diverse, children aged between 5 and 6 years old manage to interpret reflexive pronouns accurately. Their awareness of reflexive pronoun properties are detected in a line of regular mastery increase across their age; from 3 years old to 6 years old (Chien \& Wexler 1990).

The acquisition of pronouns in L1 settings is marked by the challenge that is posed by the pronominal properties within their syntactic environments. Being free referential variables, children face some difficulties in identifying that pronouns do not abide by the same syntactic restrictions that govern anaphors (Elbourne 2005). Through their performance, children exhibited a pronoun interpretation problem (PIP) (Hamann 2011). They even showed that they may not know Principle B but still treat pronouns like reflexives (Elbourne 2005). Such a fact has led to the assumption that children's preliminary abstract version of pronouns is based on their own version of anaphors, leading them to an inaccurate interpretation of pronouns in violation of Principle B (Boster 1991). The assumed pronoun interpretation problem is exemplified in the robust claim of a delay in Principle B effect (DPBE) (Conroy et al. 2009). This delay is problematic in its interpretation by claiming that the features that have to be set first are those of reflexive pronouns (Hamann 2011), by claiming that there are discourse-related features of pronouns that stipulate some pragmatic knowledge to arrive at an accurate interpretation (Chien \& Wexler 1990), and by claiming that a computational burden renders pronominal interpretation intricate for children (Reinhart 2011).

In the same vein, children in their non-reflexive pronoun interpretation select the anaphoric interpretation of pronouns in violation of Principle B as Chien and Wexler (1990) confirmed in their study. Specifically, in constructions akin to:

9. This is Mama Bear; this is Goldilocks. Is Mama Bear touching her?

Chien and Wexler (1990) report that in 50\% of the time children of 5-to-6 years old interpret the pronoun her to be anaphorically linked to its local antecedent, which violates Principle B. Being able to distinguish the biding and the coreference reading in a similar way as adults do is not yet established, based on the fact that Principle B is still being violated in constructions that allow solely the binding reading (Guasti 2012). The delay that marks children's nonreflexive pronoun interpretation is either attributed to the pragmatic nature of pronouns, when 
failing to take into consideration the context (Chien \& Wexler 1990), or to processing difficulties, when allowing a coreference reading while it is not licit (Reinhart 2006).

So far, by reference to child language research it is indicated that reflexive pronouns are set and fully acquired by children at an early age. Pronouns, per contra, remain problematic for them in processing their antecedents. Through discourse or any misidentification with reflexive pronouns, children still interpret pronouns as bound variables, in violation of Principle B. Accordingly Principle B is marked by a delay, where pronouns are mastered at a later age vis-à-vis reflexives.

\section{Reflexives and pronoun interpretation in second language research}

The same discrepancy in anaphor and pronoun acquisition is assumed also to figure in second language acquisition research. Notwithstanding the fact that second language speakers have already developed their own means of language representation (White 2003), they are still expected to do well with anaphor interpretation and to face some difficulties with pronouns. This claim is made regardless of a possibility of resorting to L1 grammar (White 2003). Adult L2 speakers, when faced with another language, may rely on processes that are already developed in their L1 system, which are susceptible to facilitate the acquisition processes of some problematic components as pronominals. Although pronoun acquisition is underresearched in L2 setting given the fact that pronouns do not allow a local antecedent across different languages, it is worth investigating whether L2 speakers face the same problems as natives in pronoun interpretation (White 2003). It is also worth studying whether the same factors such as pragmatic competence and computational capacity intervene in L2 pronoun acquisition.

Different studies addressed the acquisition of reflexive pronouns in second language settings aiming at charting the assumed parametric variations that might characterize Principle A cross-linguistically (White 1998). Two studies are considered in this regard. Thomas (1991) examined the interpretation of English anaphors by Japanese and Spanish speakers. This study is based on the hypothesis that L2 speakers do not have access to Universal Grammar (UG). This means that L2 speakers will exhibit patterns that are not part of their native language. Another hypothesis that was tested within this study stated that L2 learners could have access to UG through their native language. But any difference between the source and the target language meant that access to UG would fail. It was also tested whether L2ers could have direct access to UG through their performance in anaphor interpretation. In this study, the participants were introduced to constructions similar to Mary heard that Sue told the doctor about herself, where they had to indentify the referent of the reflexive pronoun. The experimental tasks were about eliciting pronoun interpretation of sentences and a multiplechoice task to choose a referent of a pronoun. The proficiency level was taken as an active variable in this study. The study findings showed that the 132 participants showed their awareness of the locality condition of English reflexive pronouns. Only low proficiency participants allowed long-distance binding of reflexives. These low proficiency participants were a minority in the study. Their performance was paralleled with the control group who opted for local antecedents for reflexives. The findings supported the fact that the participants did have access to UG given that they bound reflexives with a local or a long-distance 
Amani Mejri:

The interpretation of English reflexive and non-reflexive pronouns by Tunisian students

Argumentum 16 (2020), 241-259

Debreceni Egyetemi Kiadó

DOI: 10.34103/ARGUMENTUM/2020/15

antecedent. The study showed that direct access to UG is possible, since the participants could set the anaphor parameter in their L2 learning.

In the same vein of examining whether UG is accessible in second language acquisition, particularly through the acquisition of reflexives, Wakabayashi (1996) set an experiment in an effort of examining the interpretation of reflexive pronouns by Japanese speakers. The study hypothesis indicated that Japanese speakers need to reset that parameter of anaphor interpretation in order to have an accurate interpretation of English reflexives. The aim was to investigate whether the study participants had access to UG through their performance. The study participants were 40 English learners, who had started studying English at the age of 12 years old, and visited the UK for different periods of time. They were examined individually throughout the experiment. The types of the constructions introduced in the questionnaire included Tom disliked himself or Tom told Sam that the police would arrest him. The questionnaire design included every possible NP antecedent in the options associated with every construction. The findings indicated that UG is accessible to the Japanese participants. Based on their systematic accuracy with reflexives, it was shown that the participants had some linguistic knowledge to be able to interpret English reflexives (Wakabayashi 1996). This means that the informants did reset the anaphor parameter in their interpretation, which led them to a high accuracy in identifying local and long-distance antecedents in English (Wakabayashi 1996)

In their turn, pronouns did not receive the same research attention attributed to reflexives. Nevertheless, some studies addressed pronoun acquisition by focusing on specific issues. Two studies that relate in a way or another to the current study concentrate on pronoun acquisition from two perspectives: assuming that adult L2 speakers should be able to interpret pronouns given their well-developed L1 internal system (White 1998), and assuming that there is an L2 DPBE aggravated by a low proficiency factor and a computational complexity. The former study by White (1998) tested the hypothesis that L2 speakers do not face any difficulties in pronoun interpretation as adult L2ers could have recourse to their pragmatic knowledge from their L1, which is corroborated by their working memory capacity. In that, adult L2ers are supposed to manifest their Principle B knowledge. To test this hypothesis, a truth value judgement task was set to examine 13 Japanese speakers and 15 francophones (from Canada, Québec), plus an English control group. In this study, the participants had to interpret constructions based on pictures and their interpretation was acted out in a scene or linked either to a picture or a story, or both of them. Some of the test constructions included $\mathrm{Mr}$

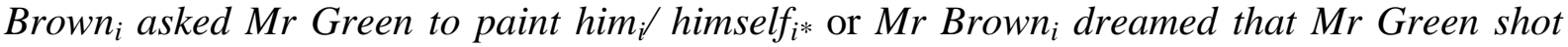
him $_{i} /$ himself $_{i *}$. The participants' performance was compared to that of an English control group. In this respect, White reports that the L2 participants exhibited a high accuracy as far as pronouns are concerned. They showed that they were aware pronouns are not bound in their local domains. White claims that adult L2ers had minor problems in pronoun resolution when compared to young L2 learners. Their pragmatic knowledge and appropriate working memory capacity helped them in interpreting pronouns. However, it is assumed that any problems adults might face in interpreting pronouns could be due to their low L2 proficiency, which is a research matter addressed by Slabakova et al. (2017).

In their study on L2 pronoun acquisition, Slabakova, White, and Guzzo (2017) hypothesized that the same difficulties facing children in interpreting pronouns face similarly L2 speakers. These difficulties are exemplified in a high computational load associated with pronouns, placing the working memory in an intricate task of computing antecedents based on 
syntactic and pragmatic requirements activated in specific environments. The experiment addressed both the full and reduced version of English pronouns. The participants were French and Spanish native speakers, plus a control group of English speakers. In a truth value judgement task, participants were supposed to encounter stories associated with some constructions where they had to indentify whether the sentences were true or false. The constructions that the participants faced followed their sitting for a proficiency test, being a version of the Oxford Test of Proficiency. It included items to test the grammatical competence of the study participants. Examples of the study constructions, on the other hand, were associated with some stories and included Harry sprayed'm or Harry sprayed him, among others. These constructions were assigned the value true or false. A false sentence would be indicative of the participants' grammatical competence because it would demonstrate the non-coreferential interpretation in accordance with Principle B. Participants' performance was accurate with the full and reduced version of pronouns, designating their high L2 proficiency as it was an operative variable in this study. Low proficiency L2 participants had an accurate performance with the reduced pronouns, but not with full ones. Low proficiency L2 speakers' performance could be compared to children's L1 performance on pronoun interpretation, given that the same process of computing antecedents is activated for both categories of population. Comparing two derivations causes a computational load that both low proficiency L2 speakers and children acquiring their L1 face in pronoun interpretation. This similarity is untenable with advanced L2 adult speakers. Therefore, this study shows a parallel that can be drawn between low proficiency L2ers and children, but equally shows the divergence in performance between advanced L2ers and children in their L1 acquisition (Slabakova et al. 2017).

\section{$3.1 \quad L 1$ vs. L2 acquisition: The case of Principle B}

While there is a research agreement on the delay that marks L1 Principle B acquisition, irrespective of its reasons, this concurrence is yet to be forged as far as second language acquisition is concerned. Variables that are considered to be operative in this respect are the configuration of the L1 internal system plus second language proficiency. These variables have led to assuming that in case of their deficiency, L2 acquisition, notably anaphora, will be identical to L1 acquisition. Such a claim needs robust research evidence toward confirming that L2ers will face the same complexities confronting children in interpreting pronouns and reflexives. In her turn, Flynn (1987) advocates that L1 and L2 acquisition are remarkably diverse given the language specific properties that signal every language. However, the acquisition enterprise for both L1 and L2 stipulates that the basic structural configuration of both languages needs to be set toward apprehending the structural attributes of anaphora in both languages. Adult L2 speakers' perception of the difference of L2 anaphor types diverges from L1 speakers, particularly in the course of acquisition. While L1 speakers are in the course of figuring the structural properties of anaphors in the language acquisition, the L2 speakers have already developed theirs, and appear to be alert to the specific properties and constraints of the L2 language system in their second language acquisition (Flynn 1987).

In respect of second language proficiency, it is acknowledged as pivotal in determining an accurate performance on pronoun interpretation in L2 acquisition. Such a case is corroborated by the study conducted by Slabakova et al. (2017), where high proficiency L2 speakers accurately interpreted English pronouns in both forms: the reduced and strong form. The L1 
interference is not considered as a potential factor shaping L2 acquisition. The dissimilarity in the pronominal systems could be captured in the current study between the Arabic and English pronominals. Arabic pronouns are composed of dependent and independent pronominal forms, given that it is a pro-drop language. The English pronouns, on the other hand, include strong and reduced pronouns. Such a pronominal distinction requires observation in the course of acquiring English as a second language.

To sum up so far, investigating complexities of pronoun interpretation remains limited in comparison with anaphor interpretation studies. Equally, research on these issues addresses languages such as Japanese, Korean, French, Spanish, among others. Yet, the Arabic language remains under-researched. For this reason, this study is elaborated to scrutinize the performance of Arabic-speaking students in interpreting English reflexive and non-reflexive pronouns. The primary aim is to gain an overall understanding of the Arabic-speaking context through this pilot study.

\section{The current study}

\subsection{The Tunisian Arabic pronominals vs. The English pronominals}

Tunisian Arabic, similar to Modern Standard Arabic, has two distinct forms of pronouns. Subject pronouns can occur as independent morphemes or as attached suffixes, whereas the object pronouns can only occur as dependent suffix pronouns. The Arabic and English object pronouns are disparate, which is exemplified in the following constructions:

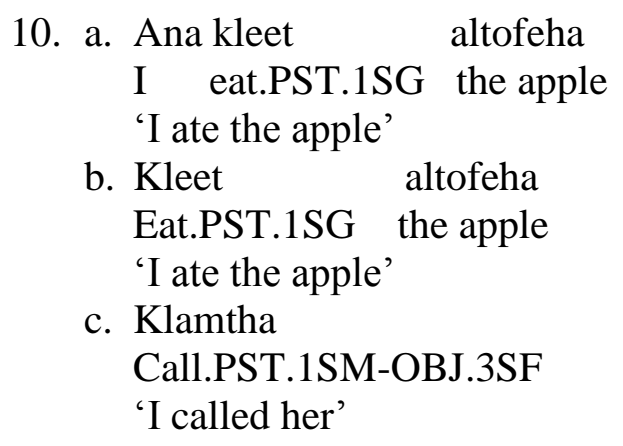

10(a) and 10(b) represent instances of independent and dependent subject pronouns in Tunisian Arabic, respectively. 10(c) shows the example of a dependent object pronoun. The verb of this sentence accommodates both the subject and object dependent pronouns. In Tunisian Arabic, as it is the case for standard Arabic, object pronouns occur only as suffixes attached to the verb or prepositions. They do not have an independent or strong form similar to the English language. Thus both sets of pronouns, the dependent as well as the independent, exhibit number and gender features. Gender features in Tunisian Arabic do not apply to the first singular and plural pronouns, the second singular and plural pronouns, and the third plural pronoun i.e., gender distinctions are only visible on third singular pronoun.

Reflexive pronouns in Tunisian Arabic are indistinguishable in construction from Modern Standard Arabic. The former is constructed by combining the word "ruh", being literally 'soul', with the possessive set of suffixes, as shown in Table 1. The latter is created by combining the term "nafs", being also literally 'soul', with the set of possessive suffixes. 
Amani Mejri:

The interpretation of English reflexive and non-reflexive pronouns by Tunisian students Argumentum 16 (2020), 241-259

Debreceni Egyetemi Kiadó

DOI: 10.34103/ARGUMENTUM/2020/15

\begin{tabular}{|c|c|c|}
\hline $\begin{array}{c}\text { The Reflexive } \\
\text { Pronouns }\end{array}$ & Features & $\begin{array}{c}\text { The English } \\
\text { Equivalent }\end{array}$ \\
\hline Ruh-i & 1 st singular & myself \\
\hline Ruh-na & 1 st plural & ourselves \\
\hline Ruh-ik & $2^{\text {nd }}$ singular & yourself \\
\hline Ruh-kum & $2^{\text {nd }}$ plural & yourself \\
\hline Ruh-u & $\begin{array}{c}\text { 3rd masculine } \\
\text { singular }\end{array}$ & himself \\
\hline Ruh-ha & 3rd feminine singular & herself \\
\hline Ruh-hum & 3rd plural & themselves \\
\hline
\end{tabular}

Table 1. The Tunisian reflexive pronouns

Reflexivization in Tunisian Arabic is expressed by using the reflexive pronouns.

11. a. Jad tawer ruh-u

Jad improve.PST.3SM himself

'Jad improved himself'

b. Amal rasmet ruh-ha

Amal paint.PST.3SF herself

'Amal painted herself'

In the two aforementioned sentences, the Tunisian reflexive pronouns are used and reflexivization is strictly expressed through their use, unlike standard Arabic where the verb morphology can include an element to express this reflexivization.

\subsection{Research aims and predictions}

This study aims at probing into a context of a language that is under-researched when compared to the English language. For this reason, in the framework of investigating pronoun interpretation in the context of the Arabic language, this piece of research aims at examining English pronoun acquisition by Tunisian learners. As it is a preliminary stage into this setting, the main focus is to explore whether there exist any differences and similarities in the interpretation of reflexive and non-reflexive pronominals for Tunisian speakers in the same pattern that native English speakers manifest when mastering reflexives earlier than pronouns. It is expected that Tunisian speakers may face the same difficulties of pronoun interpretation recorded in L1 research. This prediction is motivated by the pronominal difference between the two researched languages. It is equally motivated by the demanding nature of pronouns given that the study informants are still in the process of their L2 learning. Their L2 grammatical competence is still being forged. As it is a pilot study, neither the L2 proficiency level nor the gender variable are taken into account. Upon confirming or disconfirming any potential similarities or dissimilarities in pronoun interpretation between English and Tunisian speakers, these variables will be addressed thoroughly. 


\subsection{Participants}

24 Tunisian high school students, 6 males and 18 females, took part in this piece of research. The participants constituted a third year high school class. They pursue their studies in Tunisian public schools where they were all ushered to the English language at almost the same age. Their age ranges from 16 years old to 19 years old, as shown in table 2. They are native speakers of Tunisian Arabic. They study the French and English language in their school programs.

\begin{tabular}{|c|c|}
\hline Age Category & Number of Students \\
\hline 16 years old & 5 \\
\hline 17 years old & 16 \\
\hline 18 years old & 2 \\
\hline 19 years old & 1 \\
\hline
\end{tabular}

Table 2. The study participants' age

In the research tool of this study, participants were asked to self-evaluate their level in English language. They were given four levels from "average" to "excellent". Their self-evaluation is summarized in the following table 3.

\begin{tabular}{|c|c|}
\hline English Language Level & Subjects \\
\hline Average & 4 \\
\hline Good & 13 \\
\hline Very Good & 6 \\
\hline Excellent & 1 \\
\hline
\end{tabular}

Table 3. The participants' self-evaluation in the English language

\subsection{The questionnaire}

A questionnaire was developed for this study. It was made of three tasks, plus a final section about the participants' biographical data (gender, age, level in English, and year of exposure to English language). Each task of the questionnaire was dedicated to one anaphor type, plus the final translation task. Every construction per task addressed one type of pronouns: either a reflexive or non-reflexive pronoun. The first task addressed English pronouns. It was made up of five constructions with every construction containing one pronoun only. Each sentence had three options about the possible pronoun antecedents.

12. Mary and Kitty took a bath together. Mary washed her.
a. Her refers only to Mary.
b. Her refers only to Kitty.
c. Her refers to Mary or Kitty. 
The second task addressed reflexive pronouns. It included five constructions containing only one reflexive pronoun each. The sentences were associated with three alternatives of possible antecedents for the reflexive pronouns. Each task included more than one antecedent in the test sentences. This motive is to trigger any possible computation of the derivation associated with each anaphor.

13. Anna wanted Maria to understand herself.
a. Herself refers only to Maria.
b. Herself refers only to Anna.
c. Herself refers to Maria or Anna.

The final task was for translating constructions including reflexive and non-reflexive pronouns. The aim of this task was to detect similarities between the two languages through having participants translating the English version of the sentences into the Tunisian one. It also aimed at tracking any comprehension or miscomprehension patterns when addressing the test constructions. Examples of the translation task constructions:

14. Mark wanted Jack to introduce himself.

15. Mary needs Silvia to call her.

\subsection{Results}

\subsubsection{Pronoun interpretation}

The results obtained in this study report the participants' pronoun interpretation as summarized in table 4.

\begin{tabular}{|l|c|c|c|}
\hline \multicolumn{1}{|c|}{ Constructions } & $\begin{array}{c}\text { Option A: 1st } \\
\text { antecedent }\end{array}$ & $\begin{array}{c}\text { Option B: 2nd } \\
\text { antecedent }\end{array}$ & Option C:Both \\
\hline $\begin{array}{l}\text { Mary and Kitty took a bath } \\
\text { together. Mary washed her. }\end{array}$ & 1 & 22 & 0 \\
\hline $\begin{array}{l}\text { Mary and Kitty went to a } \\
\text { birthday party. Mary dressed } \\
\text { her. }\end{array}$ & 3 & 21 & 0 \\
\hline $\begin{array}{l}\text { Alice and Bob were playing } \\
\text { music together. Alice taught } \\
\text { him a new song. }\end{array}$ & 5 & 17 & 2 \\
\hline $\begin{array}{l}\text { Bob and Peter were playing } \\
\text { with a ball. Bob put the ball } \\
\text { near him. }\end{array}$ & 8 & 16 & 0 \\
\hline $\begin{array}{l}\text { John hated Jack's picture of } \\
\text { him. }\end{array}$ & 2 & 19 & 3 \\
\hline
\end{tabular}


The performance of the Tunisian high school students in their pronoun interpretation was marked by their accuracy with some constructions, such as (16), (17), and (18). The antecedents in these sentences do not belong to the governing categories of the pronouns with which they match.

16. Mary and Kitty took a bath together. Mary washed her.

17. Mary and Kitty went to a birthday party. Mary dressed her.

18. Alice and Bob were playing music together. Alice taught him a song.

The study participants clustered to a high degree around the antecedent that is not a potential binder within the same clause, as indicated in table 4. Construction (18), where there is a pronoun-antecedent mismatch in terms of gender, aimed at triggering a sort of computation for the students. In their interpretation, henceforth, they exhibited an awareness of this gender mismatch by eschewing the incorrect alternative (17 students out of 24 chose Bob as the antecedent of him).

Another pronoun construction that was interpreted by the Tunisian students included a pronoun within a prepositional phrase:

19. Bob and Peter were playing with a ball. Bob put the ball near him.

The preposition here is locative, indicating the place of the ball, and the pronoun is an argument within the PP. As far as this particular sentence is concerned, 16 students interpreted him as referring to Peter, whereas 8 of them thought him would refer to Bob. The rest 2 students opted for the option "both". It is relevant to note that, following Reinhart and Reuland (1993), the pronoun is a PP complement and does not belong to the matrix verb's thematic grid. As the pronoun and the antecedent are not coarguments, the pronoun intuitively is not disallowed to corefer with the subject. Accordingly, the interpretation of the Tunisian students, thus translated in selecting the antecedent with which the pronoun is not a coargument, is akin to the native interpretation.

Construction (20) received a varied performance pattern. In fact, the Tunisian students' performance was unequally distributed over the derivations associated with it.

\section{John hated Jack's picture of him.}

2 students opted for John as the potential binder, while 19 students opted for Jack as the antecedent. This construction is marked by a possessor that is considered to be the binder, provided that the anaphoric element is a reflexive pronoun, according to Binding Theory. However, in such a sentence where the anaphor is a pronoun, it is bound by the subject of the matrix verb. Yet, it can likewise be bound by the possessor with a limited degree of acceptability (Keller \& Asudeh 2001). Both anaphor types are grammatical, with a propensity of considering that reflexives are more acceptable than pronouns in this respect. The performance obtained on this construction reflects the acceptability variation that native speakers entertain towards it. Thus, as the Tunisian students also manifested, it is possible to accept, to some extent, that a pronoun is bound by the possessor. 


\subsubsection{Reflexive pronoun interpretation}

Concerning the participants' interpretation of the English reflexive pronouns, it could be considered as a varied performance, as demonstrated in table 5.

\begin{tabular}{|l|c|c|c|}
\hline \multicolumn{1}{|c|}{ Constructions } & $\begin{array}{c}\text { Option A: 1st } \\
\text { antecedent }\end{array}$ & $\begin{array}{c}\text { Option B: 2nd } \\
\text { antecedent }\end{array}$ & $\begin{array}{c}\text { Option C: } \\
\text { Both }\end{array}$ \\
\hline $\begin{array}{l}\text { Tom showed Bill a picture of } \\
\text { himself. }\end{array}$ & 15 & 6 & 3 \\
\hline Mary talked to Alice about herself. & 21 & 3 & 0 \\
\hline $\begin{array}{l}\text { Peter and Jack were wet. Jack } \\
\text { dried himself. }\end{array}$ & 9 & 15 & 2 \\
\hline $\begin{array}{l}\text { Anna wanted Maria to understand } \\
\text { herself. }\end{array}$ & 4 & 18 & 2 \\
\hline $\begin{array}{l}\text { Bill wanted John to understand } \\
\text { himself. }\end{array}$ & 12 & 11 & 1 \\
\hline
\end{tabular}

Table 5. The participants' interpretation of reflexive pronouns

A construction that is considered critical even for native speakers encompasses a PNP (21):

21. Tom showed Bill a picture of himself.

15 of the participants indicated that the convenient antecedent for the reflexive is Tom, while 6 of them designated Bill as the antecedent. The remaining 3 students were inclined for "both" NPs as the antecedents, as indicated in table 5. Another accurate interpretation is detected in construction (22):

\section{Mary talked to Alice about herself.}

The informants here, specifically 21 of them, opted for Mary as the antecedent of the reflexive, while 3 students chose Alice as the antecedent. It is crucial to note that this construction is an instance of an exempt anaphor. To elucidate, the antecedent and the reflexive pronoun do not hold thematic roles with the matrix verb. Rather, the reflexive pronoun is part of the PP that is an adjunct of the verb. The anaphor is not precisely a reflexive realization, but an exemplification of an exempt anaphor, being discourse-based rather than syntactically governed. That is the anaphor is the complement of the preposition that forms a semantic predicate and its interpretation is logophoric since there is no local binding.

The task channelled to the reflexive pronouns included two other constructions where anaphors are part of infinitival clauses:

23. Anna wanted Maria to understand herself.

24. Bill wanted John to understand himself. 
The participants' interpretation of the reflexive pronouns in these sentences could be considered unequal due to the distribution of their performance. For sentence (23), 18 of the participants opted for Maria as the appropriate antecedent, while 4 of them selected Anna as the antecedent of herself. However, in construction (24), 11 of the Tunisian participants determined John as the favourable antecedent, whereas 12 of them decided that Bill is the suitable antecedent. The non-consistency in these two constructions may allude to some chance performance among the participants.

In constructions that are straightforward in respect of reflexivity (25), the Tunisian students were somehow accurate in their performance.

\section{Peter and Jack were wet. Jack dried himself.}

15 students selected Jack as the antecedent of the reflexive pronoun himself, while 9 students inaccurately interpreted this construction by selecting Peter as the antecedent. The rest 2 students chose the option "both" (Peter and Jack) as the antecedent.

The translation task, being the final one encompassing English constructions to be translated into the Tunisian language, reflects an interesting pattern in the performance of the study informants. They were meant to interpret two anaphora types in the four constructions:

26. Anna always talks to Silvia about herself.

27. Peter and Bob were playing with a ball. Bob put the ball near him.

28. Mark wanted John to understand himself.

29. Mary needs Silvia to call her.

Constructions (26) and (28) encompass reflexive pronouns. Based on the translation of the informants, it would be possible to retrieve more information on their interpretation. Regarding the reflexive pronouns, 13 and 14 study participants had an accurate translation of constructions (26) as well as (29), respectively. These figures, when compared to values amassed from task 2, could be seen as matching to some extent. The same distribution of the participants' interpretation, translated into numerical figures, in task 2 could equally be detected in the translation task, but still leads to questioning this sort of performance. However, taking into account this performance alludes to considering the sort of errors that marked the way the Tunisian students approached these constructions. The reiterated errors in the translation were principally being unable to comprehend the construction, such as translating (26) as "Anna knows Silvia", "Anna wants to talk about herself" (overlooking the NP Silvia), or "Anna goes to Silvia", as well as matching the reflexive with the inappropriate antecedent, such as translating (28) as "Mark wants to introduce himself" or "Mark introduces himself to Jack".

In constructions (27) as well as (29), the participants encountered pronouns. 15 and 12 of the Tunisian students had faultless translations of the constructions (27) and (29), respectively. In an attempt to compare these figures to the ones retrieved from the first task tackling pronouns, it could be observed that the participants' accuracy with pronouns interpretation is limited. Such a fact leads to diagnosing the error types that emerged in their translation. Some of these errors comprised misunderstanding the constructions, such as translating sentence (27) as "Bob and Peter were playing with a ball", "Bob scored a goal against them", and gender mismatch, such as translating sentence (29) as "Mary needs Silvia 
to talk to him", or "Mary needs Silvia to introduce himself". The same errors that marked the reflexive constructions approximately marked the pronoun constructions in the translation task.

\subsection{Discussion}

As far as pronoun interpretation is concerned, the Tunisian participants displayed their knowledge of the structural properties that govern pronouns. Their interpretation of constructions (16), (17), as well as (18) conveys their awareness that pronouns are free within their local domains. While constructions (16) and (17) do not incorporate any gender mismatch that is susceptible to enhance the computational load of pronoun interpretation, construction (18) deliberately involves an instance of gender mismatch. The aim was to distract the participants with two candidate antecedents. It was expected that this construction would place a particular difficulty for the study informants and it would lead the informants to misinterpret the pronoun. Surprisingly, a high number of them clustered around the accurate antecedent for the pronoun. Such a fact hinges upon the participants' awareness of the structural properties of pronouns, even though the gender mismatch did not maximize the students' performance. Unlike the finding of Chien and Wexler (1990) that indicates the gender mismatch helped the participants opt for the appropriate pronoun antecedent, in this piece of research gender helped, but only to a limited extent, the participants select the licit antecedent. It may be seen as a lack of attention to the properties of the pronoun, hence pronoun interpretation is this regard might be systematic or the outcome of chance for the students.

In fact, this task encompasses constructions that are critical according to some studies (Keller \& Asudeh 2001). These sentences are (20), including a PNP, and (19), comprising a PP. As far as the PNP is concerned, it was perceptible that the study participants hesitated in their antecedent choice. It followed from the fact of having two potential binders: the matrix subject and the possessor. According to binding theory, a reflexive pronoun is the grammatical choice given that the binder is the possessor. However, as Keller and Asudeh claim, a pronoun could equally be accepted to some extent, but with a high propensity of accepting reflexives. In light of this claim, 2 of the participants accepted the possessor as the binder, while 19 of the students selected the matrix subject as the antecedent. This performance pattern is similar to that of the native speakers in the study of Keller and Asudeh (2001). A confined number accepted the possessor as the antecedent, while the highest number of their participants accepted the subject of the matrix verb as the antecedent. Such a fact could provide an insight on the joint patterns that punctuate pronoun interpretation in L1 and L2 contexts.

The reflexive pronouns task, per contra, was devoid of any gender mismatch, unlike pronouns. The aims was to provide constructions similar to the ones proposed in L1 studies and to examine whether reflexives, without any bias or inherent difficulties of any possible mismatch, would be problematic for the Tunisian students. The participants did not have to compute any potential antecedents based on gender congruity with the reflexives. Yet, their reflexive interpretation is telling in the way they approached them. Some of the reflexive pronouns in this task abide by Condition A, while other reflexive pronouns are exempt from the syntactic properties of binding in the local domain. The participants encountered some exempt anaphors that are discourse-oriented. Their performance on the former sort of reflexives characterized their knowledge of the locality condition where anaphors have to be bound. When met with constructions where the reflexive is part of a PP, the informants 
Amani Mejri:

The interpretation of English reflexive and non-reflexive pronouns by Tunisian students

Argumentum 16 (2020), 241-259

Debreceni Egyetemi Kiadó

DOI: 10.34103/ARGUMENTUM/2020/15

considered the reflexive pronoun as an exempt anaphor that is bound by the matrix subject. Their interpretation could be discourse-based, given that they have already developed their L1 pragmatic competence and could be sensitive to the impact of discourse in this anaphor type. In constructions where the reflexives are part of ECMs, the participants appeared to be divided to two almost unequal groups. The two prospective antecedents were both selected by a high number of the participants as indicated previously. This type of construction posed a specific challenge for the students. With the other sort of constructions, they tended to cluster around the equitable antecedent. With infinite clauses they seemed hesitant on the adequate antecedent. However, it is still doubted whether this particular performance is the accurate characterisation of the participants' grammatical competence or the outcome of some chance interpretation, especially as the proficiency level and the gender factor are not taken as operative variables in this study. For natives, in fact, the nature of these constructions places a challenge too. The antecedent of reflexives in such constructions is the subject of the ECM that receives exceptional case from the matrix verb (Reihart \& Reuland 1993). Such a structural complexity exhausts the ability of native speakers, as well as that of L2ers, to be accurate in their interpretation.

Another critical structure that the participants encountered in this study was the PNP construction, devoid of any possessor. As most of the study informants opted for the complement of the verb as the antecedent rather than the matrix subject, it could be entertained that, akin to native speakers, they had the propensity of binding the reflexive with the possessor, if available, or with the NP that held the syntactic position of a possessor. This finding resonates with the findings of Keller and Asudeh (2001) in demonstrating the acceptability of reflexives and pronouns in these contexts, the binding options available for the speakers, and the structural constraints they have to observe. Based on the obtained findings, the participants exhibited that some syntactic constraints burdened their interpretation. Although they manifested some comprehension matters when encountered with some constructions in the translation task, no pragmatic deficiency could be diagnosed through this piece of datum. Such a fact is in line with White (1998) who proposes that L2ers might not have pragmatic challenges based on their already developed L1 systems. Yet to properly draw such a conclusion, it is crucial to directly address the second language proficiency level as an operative variable in pronoun interpretation.

Some hypotheses of this study are inspired from other L2 studies claiming that the same delay in Principle B facing L1 children is also characteristic of L2 acquisition (Slabakova et al. 2017). Yet to prove that Principle B delay is part of L2 acquisition, it would be a proviso to alter the methodology, broaden the population by having equal numbers of male and female participants, and to address L2 proficiency. White (1998) postulates that L2ers do not necessarily face the same delay in pronoun acquisition thanks to their L1 internal language system. Such a claim needs a particular attention attributed to the participants' L1 interference and L1 pronominal system. Arabic pronominals, in this respect, are different from their English equivalents. Advocating that Arabic, as an L1, might have influenced the way the participants interpreted the English pronouns could not be tenable through this study. This is based on the fact that the Tunisian participants might have their own strategies in their Arabic pronoun interpretation that cannot be transferred to the task of English pronoun interpretation. Yet, a confirmatory study needs to be elaborated in this regard. Firstly, the methodology endorsed in this pilot study does not entirely capture the Arabic setting where the English language is acquired. It only outlines the performance patterns of anaphor interpretation in a particular L2 
setting regardless of L2 proficiency and gender distribution. Secondly, examining the strategies that Tunisian speakers might have developed to interpret Arabic pronouns could be informative in the process of examining their L2 pronoun interpretation. Therefore, the question of second language acquisition in a different linguistic, cognitive, and social condition (Slabakova et al. 2017) stipulates that the observed pattern in an L2 setting needs to be accounted for in terms of L1 strategies and the possible interference of the gender variable, plus the importance of the L2 proficiency in addition to using different methodological tools.

\section{Conclusion}

In this study, the investigation of reflexive and non-reflexive pronoun interpretation was conducted in an Arabic language context, particularly Tunisian Arabic. The aim was to gain an initial view on this context of an under-researched language. Similarly, the study had a focus of exploring any possible similarities and differences in the interpretation of English pronouns by Tunisian learners when compared to some studies on English child language acquisition. To this end, a questionnaire was developed addressing the acquisition of reflexive and non-reflexive pronouns. Specifically, the participants of this pilot study, being mainly Tunisian high school learners, had to interpret some pronouns in different types of constructions such as picture NPs, PPs, ECMs, among others. Their performance as far as pronouns are concerned was marked by some sort of variation. They opted most of the time for the appropriate antecedent choice. In their reflexive pronoun interpretation, they were equally accurate but with a higher degree, but still within a pattern of variation. In spite of being accurate most of the time in the interpretation tasks, the translation task showed that the participants did have some comprehension problems that led them to misunderstanding, hence inaccurately translating some constructions. Variables such as language proficiency and gender were not taken as operative factors in this introductory piece of research. For this reason, only a global introduction was provided through this study regarding the context where English is a second language and Arabic is the native language. However, to confirm or disconfirm that the obtained performance is indicative of the participants' L2 grammatical competence still needs further research within the same context, by relying mainly on different methodology, L2 proficiency, and the effect of gender distribution.

\section{References}

Boster, C.T. (1991): Children's failure to obey Principle B: Syntactic problem or lexical error. Ms., University of Connecticut, Storrs.

Chomsky, N. (1986): Knowledge of Language: Its Nature, Origin and Use. New York: Praeger. https://doi.org/10.1016/0388-0001(89)90029-6

Chien, Y-C., \& Wexler, K. (1990): Children's knowledge of locality conditions in binding as evidence for the modularity of syntax and pragmatics. Language acquisition 1.3, 225-295. https://doi.org/10.1207/s15327817la0103_2

Conroy, A., Takahashi, E., Lidz, J., \& Phillips, C. (2009): Equal treatment for all antecedents: How children succeed with Principle B. Linguistic Inquiry 40(3), 446-486. https://doi.org/10.1162/ling.2009.40.3.446 
Elbourne, P. (2005): On the acquisition of Principle B. Linguistic inquiry 36.3, 333-365. https://doi.org/10.1162/0024389054396908

Elbourne, P. (2003): Are our children speaking Middle English. CUNY Syntax Supper.

Flynn, S. (1987): A parameter-setting model of L2 acquisition: Experimental studies in anaphora. Reidel Publishing Company: Netherlands. https://doi.org/10.1007/978-94-009$\underline{3747-5}$

Grodzinsky, Y., \& Reinhart, T. (1993): The innateness of binding and coreference. Linguistic inquiry 24.1, 69-101.

Guasti, T. M. (2012): Language acquisition: the growth of grammar. Cambridge (MA): MIT Press.

Haegeman, L. (1994): Introduction to government and binding theory. Oxford: WileyBlackwell.

Hamann, C. (2001): Binding and coreference: Views from child language. In: Handbook of generative approaches to language acquisition, 247-290. Dordrecht: Springer. https://doi.org/10.1007/978-94-007-1688-9_7

Keller, F., \& Asudeh, A. (2001): Constraints on linguistic coreference: Structural vs. pragmatic factors. In: Proceedings of the Annual Meeting of the Cognitive Science Society, 23.23.

Reinhart, T. (2011): Processing or pragmatics? Explaining the coreference delay. The processing and acquisition of reference, 157-94.

https://doi.org/10.7551/mitpress/9780262015127.003.0007

Reinhart, T. (2006): Interface strategies. Cambridge, MA: MIT Press. https://doi.org/10.7551/mitpress/3846.001.0001

Reinhart, T., \& Eric, R. (1993): Reflexivity. Linguistic inquiry 24.4, 657-720.

Slabakova, R., Lydia W., \& Natália B.G. (2017): Pronoun interpretation in the second language: Effects of computational complexity. Frontiers in psychology 8, 12-36. https://doi.org/10.3389/fpsyg.2017.01236

Thomas, M. (1995): Acquisition of the Japanese reflexive zibun and movement of anaphors in Logical Form. Second Language Research 11.3, 206-234.

https://doi.org/10.1177/026765839501100302

Thornton, R., \& Wexler, K. (1999): Principle B, VP ellipsis, and interpretation in child grammar. Cambridge (MA): MIT Press. https://doi.org/10.7551/mitpress/5550.001.0001

Wakabayashi, S. (1996): The nature of interlanguage: SLA of English reflexives. Second Language Research 12.3, 266-303. https://doi.org/10.1177/026765839601200302

White, L. (2003): Second language acquisition and universal grammar. Cambridge: Cambridge University Press.

White, L. (1998): Second language acquisition and Binding Principle B: child/adult differences. Second language research, 14,4, 425-439. https://doi.org/10.1017/CBO9780511815065

\author{
Amani Mejri \\ University of Debrecen, Doctoral School of Linguistics \\ University of Debrecen, Institute of English and American Studies \\ Pf. 400 \\ H-4002 Debrecen \\ amany.mejri@gmail.com
}

